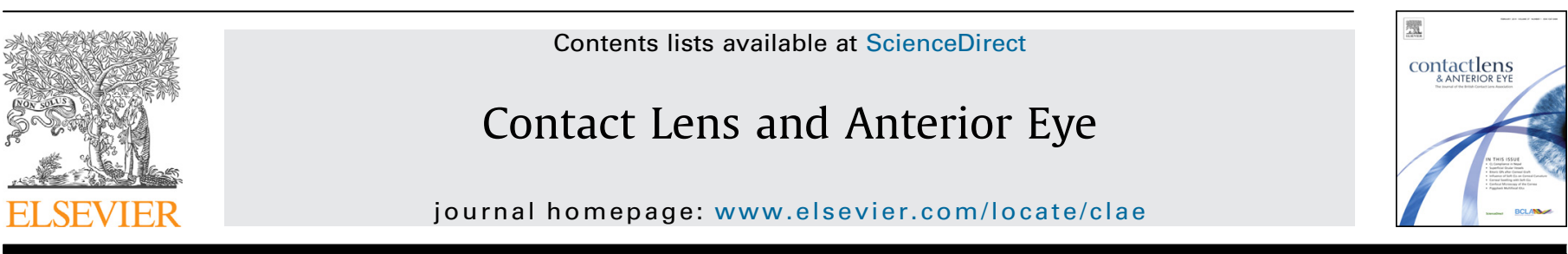

Case report

\title{
Stabilization in early adult-onset myopia with corneal refractive therapy
}

\author{
José M. González-Méijome*, Gonzalo Carracedo, Daniela Lopes-Ferreira, \\ Miguel A. Faria-Ribeiro, Sofia C. Peixoto-de-Matos, António Queirós \\ Clinical and Experimental Optometry Research Lab. Center of Physics, University of Minho, Braga, Portugal
}

\section{A R T I C L E I N F O}

\section{Article history:}

Received 22 December 2014

Received in revised form 2 June 2015

Accepted 30 June 2015

\section{Keywords:}

Orthokeratology

Corneal refractive therapy

Myopia control

Early adult-onset myopia

\begin{abstract}
A B S T R A C T
Purpose: To describe the stabilization of early adult-onset myopia in three university students after initiating orthokeratology treatment with corneal refractive therapy contact lenses.

Methods: Three Caucasian early adult-onset progressing myopic subjects ( 1 male, 2 females) were fitted with corneal refractive therapy lenses to correct myopia between -1.50 and $-2.50 \mathrm{D}$ of sphere using Paragon CRT (Paragon Vision Sciences, Mesa, AZ) lenses for overnight orthokeratology. The pre-treatment refractive history from 2005 as well as refraction and axial length after treatment onset are reported over a period of 3 years between December 2009 and January 2013 with an additional year of follow-up after treatment discontinuation (January-December 2013). The peripheral refractive patterns and topographic changes are also reported individually.

Results: Treatment was successful in all three subjects achieving uncorrected visual acuity of 20/20 or better monocularly. During a period of 3 years of follow-up the subjects did not experience progression in their refractive error, nor in their axial length (measured during the last 2 years of treatment and 1 year after discontinuation). Furthermore, the subjects recovered to their baseline refraction and did not progressed further over the following year after lens wear discontinuation.

Conclusions: We cannot attribute a causative effect to the orthokeratology treatment alone as underlying mechanism for myopia stabilization in this 3 patients. However, the present report points to the possibility of stabilization of early adult-onset myopia progression in young adults using corneal refractive therapy treatment.
\end{abstract}

(c) 2015 British Contact Lens Association. Published by Elsevier Ltd. All rights reserved.

\section{Introduction}

Early onset myopia is the primary condition indicated for myopia control treatments involving different strategies. Children with myopia experience a faster rate of myopia progression between 8 and 12 years of age [1] and commonly progress at lower rates during adolescence and adulthood. Furthermore, there is evidence that younger children may progress in nearsightedness as a function of the time spent reading [2,3]. As a consequence, childhood-onset myopia presents a greater risk for higher dioptric values and a worse prognosis for potential co-morbidities in adulthood [4].

Early adult-onset myopia is regarded as being a more benign condition since it commences later in life and progresses at slower rates $[5,6]$, and reaches lower final degrees of myopia compared

\footnotetext{
* Corresponding author.

E-mail address: jgmeijome@fisica.uminho.pt (J.M. González-Méijome).
}

with childhood-onset myopia. These patients also show altered accommodative and binocular function compared to age matched emmetropes [7]. Contrary to childhood-onset myopia, early adultonset myopia rarely achieves values above -5.00 or -6.00 diopters of myopia which are generally associated with pathological myopia [8]. Despite this, eyes with lower values of myopia are also at significant risk of developing several pathological complications that might compromise visual function such as accommodative dysfunction and retinal pathology [3]. This is a matter of concern because of the potential future sequelae which includes severe retinal disease.

Corneal refractive therapy has reinforced its role as a viable option to correct low-to-moderate myopia during the last 10 years [9]. Corneal refractive therapy has proved to be efficacious to slow myopia progression in children in at least 5 different peer reviewed and published studies around the world [10-15], including one controlled randomized clinical trial [16-18]. However, these studies have been conducted only in early onset myopia. However, it might be the case that progression of adult-onset myopic could 
be susceptible to be reduced using orthokeratology, based on the same working principles of inducing relative peripheral myopic refraction.

The purpose of this case series of three individuals is to report axial and peripheral refraction, corneal topography and axial length over a period of four years after being fitted with corneal refractive therapy lenses.

\section{Case reports}

The three subjects were university students participating in a study involving myopia correction with corneal refractive therapy (Paragon CRT, Mesa, AZ) and decided to remain in this modality of visual correction after the study had finished by December 2009 [19]. They underwent yearly examinations thereafter between January 2010 and December 2012 comprising three years of corneal refractive therapy treatment and one year after lens discontinuation between January and December 2013, including non-cycloplegic refraction, corneal topography (Medmont E300, Australia) and axial length (IOL Master, Zeiss, Jena). All procedures at baseline as well as during follow-up were conducted by the same clinician and five repeated measures of biometry and three repeated measures of corneal topography were obtained at each follow-up visit. By recalling their old prescriptions (based on noncycloplegic refraction as reported by them), we could verify that they were first corrected for very low myopia when they were
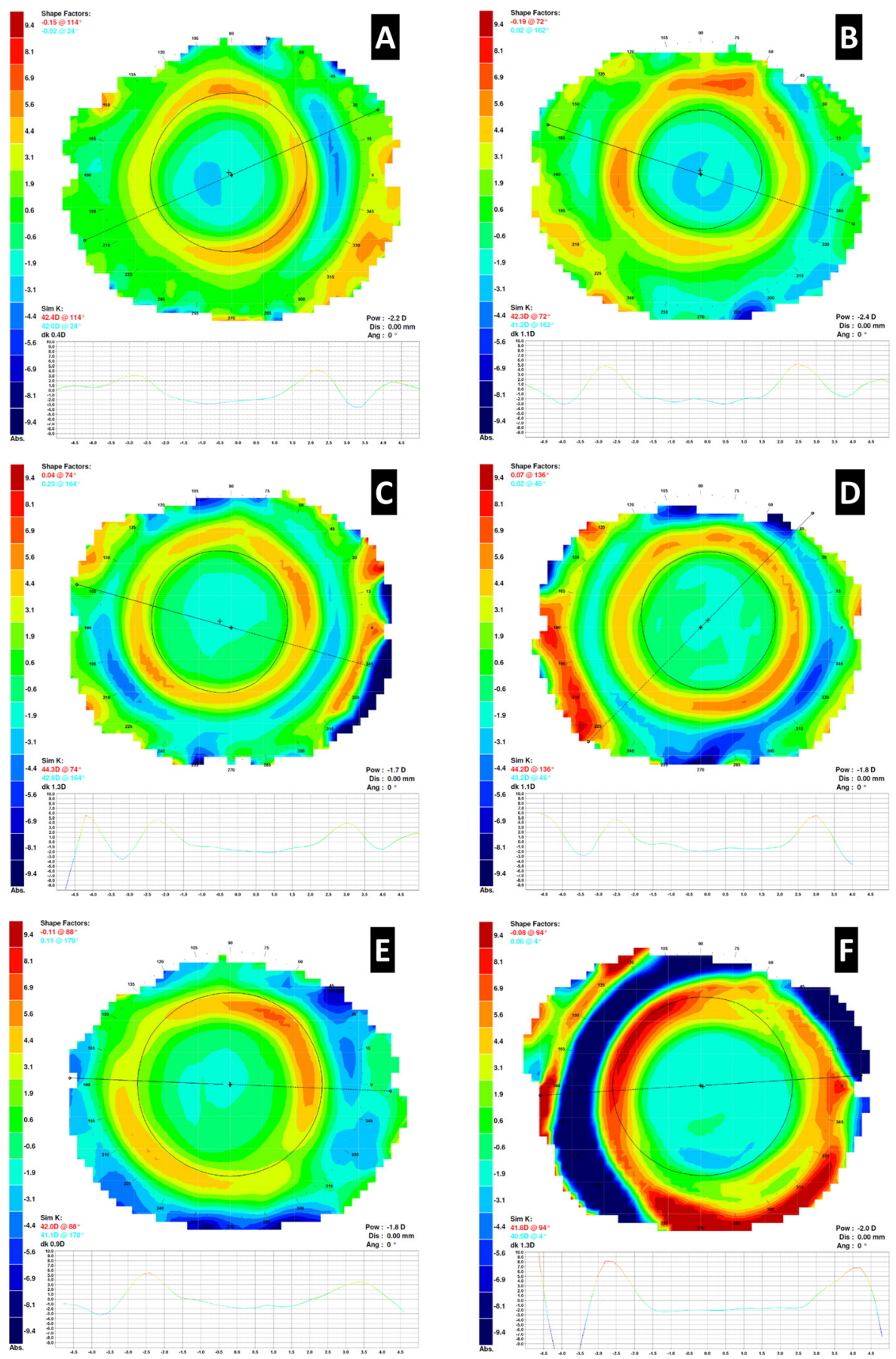

Fig. 1. Differential tangential curvature maps depicting the treatment zone of right eyes (A,C, E) and left eyes (B, D, F) for subject \#1 (A, B), \#2 (C, D) and \#3 (E, F).

Please cite this article in press as: J.M. González-Méijome, et al., Stabilization in early adult-onset myopia with corneal refractive therapy, Contact Lens \& Anterior Eye (2015), http://dx.doi.org/10.1016/j.clae.2015.06.009 
between 17 and 21 years of age. This places them in the category of early adult-onset myopia [20]. Also common to them was the fact that their myopia had progressed by $0.50 \mathrm{D}$ or more in at least one eye during the year before study commencement. This fact was an additional motivation for them to participate in the study as they had been aware on the potential benefit of corneal refractive therapy for regulating myopia progression. They were informed that the study was not intended to demonstrate the regulation of their progression. None of them had used contact lenses before. In addition to a full optometric examination including lensometry of their habitual spectacle correction, the refractive history from the first manifestation of myopia was collected through the written prescriptions they or their parents had kept over the years. Lenses were fitted according to the recommendations of the manufacturer and procedures including corneal topography and fluorescein assessment of diagnostic lenses were performed according to previously published protocols $[19,21]$. Fig. 1 shows the pretreatment and post-treatment topographical results for the 6 eyes presented in this report.

Table 1 presents the demographic information along with the refraction over the period of follow-up as well as optical biometric data obtained with IOLMaster (Zeiss, Germany) during the last 2 years of follow-up with the CRT treatment plus the additional year of follow-up after lens discontinuation. Five repeated measures were obtained at each examination. The non-cycloplegic refractive information available reports back to 2001 in one case and to 2004 in the other two cases when the onset of myopia was presumed to take place according to the prescriptions recalled from them and their parents. All three subjects were spectacles wearers and had updated their prescription yearly or every two years from 2004 to 2009. Their progression was symmetric in both eyes showing an increase of $-1.25,-0.75$ and -1.75 for subject $\# 1$, \#2, and \#3, respectively over the four year period between 2005 and 2009. At the time of recruitment in the original study, their axial length was not measured and this measure was only obtained from December 2009 and after. Axial length measurements showed small fluctuations with a trend to increase overtime during the 4 years of follow-up. The accumulated changes in axial length averaged $0.12 \pm 0.03 \mathrm{~mm}$ ranging from 0.08 for the right eye of subject \#2 and 0.15 for the right eye of subject \#1. By 2012, the three subjects discontinued the treatment as they were moving out of the university to undergo post-graduate studies at other institutions and were evaluated one year later in 2013. Corneal curvature recovered to the original topographic pattern and the original Sim$\mathrm{K}$ readings after discontinuation with differences in apical radius and Sim-K values $\leq 0.05 \mathrm{~mm}$. Fig. 2 shows the patterns of relative peripheral refractive error obtained with an open-field autorefractometer following the procedures previously published [22$23]$. Data presented report the sagittal $\left(F_{\mathrm{S}}\right)$ and tangential $\left(F_{\mathrm{T}}\right)$ astigmatic focal lengths for each eye of each subject, before and after treatment [24-25]. It is observed that two subjects had a significant peripheral hyperopic sagittal focal length before the treatment becoming emmetropic for the $70^{\circ}$ of field during orthokeratology treatment. The tangential focal length was emmetropic or slightly myopic before the treatment and became much more myopic during treatment.

\section{Discussion}

This case series describes the clinical course of three myopes with progressive, early adult-onset myopia whose refractive error stabilized after initiation of corneal refractive therapy treatment.

Table 1

Refractive history from presumed myopia onset, demographic, manifest non-cycloplegic refraction and biometric data available from the three subjects.

\begin{tabular}{|c|c|c|c|c|c|c|c|}
\hline & \multirow[b]{2}{*}{ Gender } & \multirow[b]{2}{*}{$\begin{array}{l}\text { Age } \\
\text { (by 2008) }\end{array}$} & \multirow[b]{2}{*}{ History \& follow-up } & \multicolumn{2}{|l|}{ Right eye } & \multicolumn{2}{|l|}{ Left eye } \\
\hline & & & & Refractive history & Axial length & Refractive history & Axial length \\
\hline Subject \#1 & Male & $\begin{array}{l}27 \\
\text { Presumed myopia onset:21 }\end{array}$ & $\begin{array}{l}2002 \text { (onset) } \\
2005 \\
2006 \\
2007 \\
2008 \\
2009\left(^{*}\right) \\
2010 \\
2011 \\
2012\left(^{* *}\right) \\
2013\end{array}$ & $\begin{array}{l}-0.25 \\
-1.00-0.25 \times 165^{\circ} \\
-1.50-0.25 \times 155^{\circ} \\
(---) \\
-2.00-0.25 \times 160^{\circ} \\
-2.25-0.25 \times 160^{\circ} \\
\text { Plano } \\
\text { Plano } \\
\text { Plano } \\
-2.50-0.50 \times 155^{\circ}\end{array}$ & $\begin{array}{l}- \\
- \\
- \\
- \\
- \\
25.53 \\
25.57 \\
(---) \\
25.68\end{array}$ & & $\begin{array}{l}- \\
- \\
- \\
- \\
- \\
25.52 \\
25.55 \\
(--) \\
25.63\end{array}$ \\
\hline Subject \#2 & Female & $\begin{array}{l}23 \\
\text { Presumed Myopia Onset:17 }\end{array}$ & $\begin{array}{l}2004 \text { (onset) } \\
2005 \\
2006 \\
2007 \\
2008 \\
2009\left(^{*}\right) \\
2010 \\
2011 \\
2012\left(^{* *}\right) \\
2013\end{array}$ & $\begin{array}{l}-0.50-0.50 \times 160^{\circ} \\
-1.50-0.75 \times 160^{\circ} \\
-1.50-1.00 \times 155^{\circ} \\
(--) \\
-1.75-1.00 \times 150^{\circ} \\
-2.25-1.00 \times 160^{\circ} \\
\text { Plano } \\
\text { Plano } \\
\text { Plano } \\
-2.50-1.25 \times 160^{\circ}\end{array}$ & $\begin{array}{l}- \\
- \\
- \\
- \\
- \\
- \\
24.58 \\
24.63 \\
(---) \\
24.66\end{array}$ & $\begin{array}{l}-0.25-0.50 \times 60^{\circ} \\
-1.25-0.75 \times 60^{\circ} \\
-1.50-0.75 \times 55^{\circ} \\
(---) \\
-1.50-1.00 \times 60^{\circ} \\
-2.00-1.00 \times 60^{\circ} \\
\text { Plano } \\
\text { Plano } \\
\text { Plano } \\
-2.25-1.00 \times 60^{\circ}\end{array}$ & $\begin{array}{l}- \\
- \\
- \\
- \\
- \\
24.46 \\
24.49 \\
(--) \\
24.59\end{array}$ \\
\hline Subject \#3 & Female & $\begin{array}{l}25 \\
\text { Presumed Myopia Onset: } 21\end{array}$ & $\begin{array}{l}2004 \text { (onset) } \\
2005 \\
2006 \\
2007 \\
2008 \\
2009\left(^{*}\right) \\
2010 \\
2011 \\
2012\left(^{* *}\right) \\
2013\end{array}$ & $\begin{array}{l}-0.25-0.25 \times 170^{\circ} \\
-0.50-0.50 \times 120^{\circ} \\
-1.00-0.50 \times 120^{\circ} \\
-1.25-0.25 \times 180^{\circ} \\
-1.50-0.25 \times 165^{\circ} \\
-1.75-0.50 \times 165^{\circ} \\
\text { Plano } \\
\text { Plano } \\
\text { Plano } \\
-2.00-0.50 \times 160^{\circ}\end{array}$ & $\begin{array}{l}- \\
- \\
- \\
- \\
- \\
24.35 \\
24.40 \\
(---) \\
24.48\end{array}$ & $\begin{array}{l}-0.25-0.25 \times 170^{\circ} \\
-0.50-0.25 \times 180^{\circ} \\
-1.00-0.25 \times 145^{\circ} \\
-1.50-0.25 \times 180^{\circ} \\
-1.50-0.25 \times 165^{\circ} \\
-2.00-0.25 \times 165^{\circ} \\
\text { Plano } \\
\text { Plano } \\
\text { Plano } \\
-2.25-0.50 \times 175^{\circ}\end{array}$ & $\begin{array}{l}- \\
- \\
- \\
- \\
- \\
24.54 \\
24.57 \\
(--) \\
24.66\end{array}$ \\
\hline
\end{tabular}

$\left({ }^{*}\right)$ Corneal refractive therapy (CRT) initiation: 2008; $\left(^{* *}\right)$ CRT discontinuation: 2012; (---): No data available. 
A

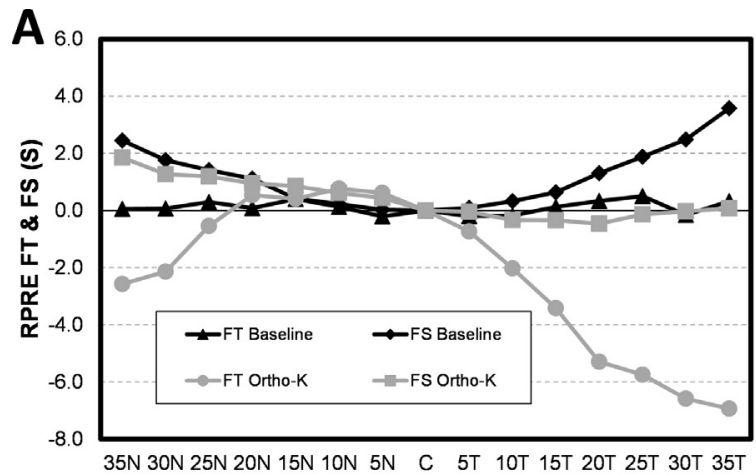

C

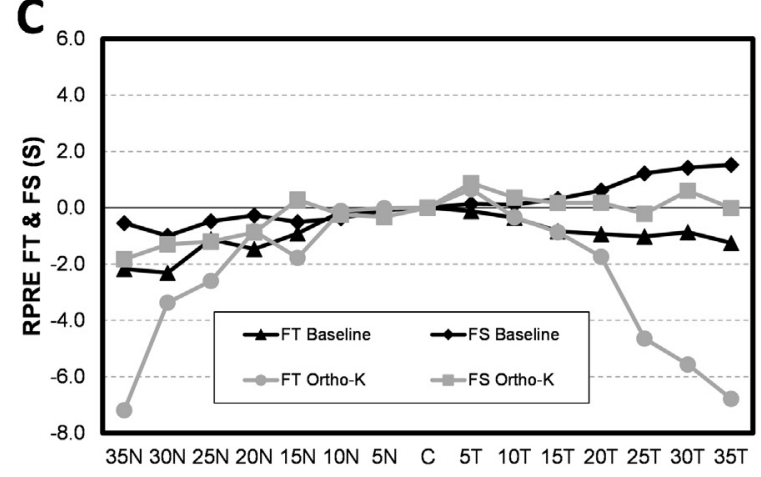

E

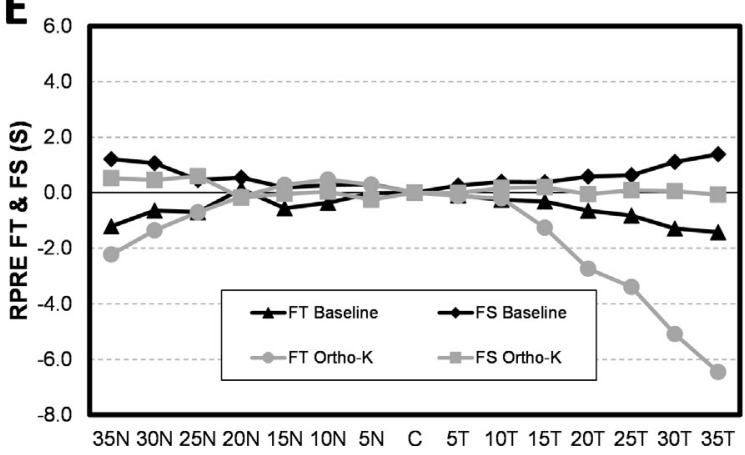

B

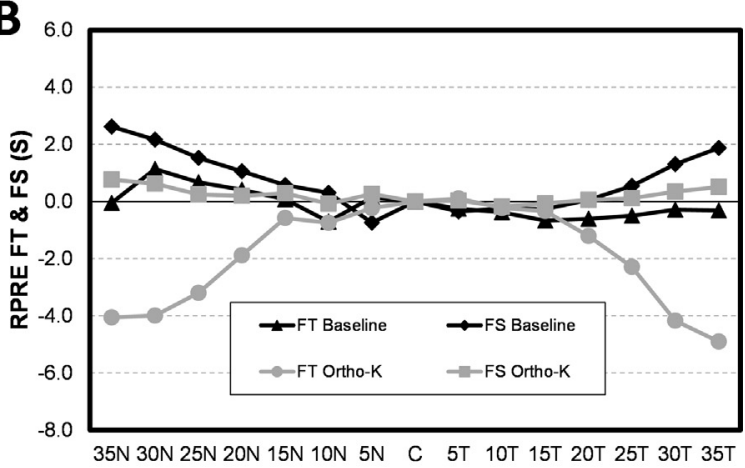

D

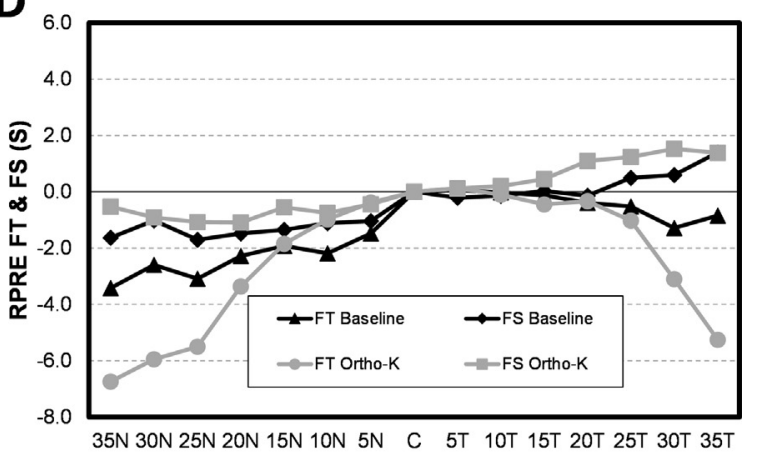

$\mathbf{F}$

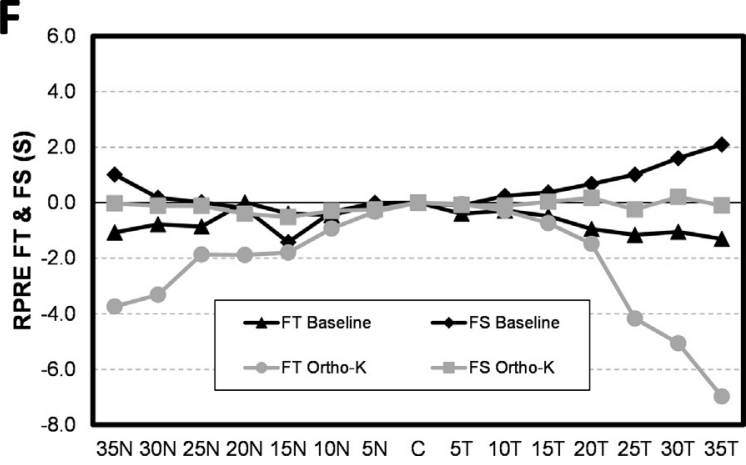

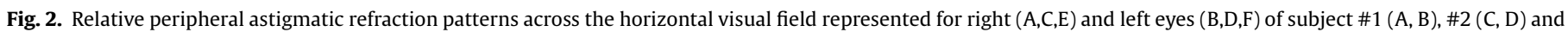
\#3 (E, F).

Stable subjective refractions were evident in each subject over two years of treatment and with minor changes after treatment discontinuation. Based on the refractive history, those subjects showed a typical pattern of early adult-onset myopia progression [8]. Considering the efficacy of orthokeratology in slowing myopia progression in younger myopes, it might be argue that a similar process might be expected in older myopes, considering that the corneal changes would produce a similar peripheral refractive effect that is supposed to be related with the myopia regulation effect [26].

Even if we assume that myopia has not progressed after lens fitting, we cannot ensure that this is because of the corneal refractive therapy treatment itself. An alternative explanation might be found in a natural stabilization of these eyes with myopia. Moreover, assuming that the initiation of the university period was associated with the onset of myopia as a consequence of an increased near-work demand, a decrease in such activities will result in a stabilization of progression. However, we cannot justify a change in the pattern of myopia progression after CRT lens fitting with a change in near work or other potential confounding factors. Indeed, it is important to highlight that over the 5 years of followup at our facilities the students remained with similar or increased loads of near work due to accomplishment of undergraduate and
MSc or PhD programs. According to the natural course of myopia progression in adolescents and young adults we could hypothesize that these subjects would probably continue progressing if left in spectacles after 2009. The work of Grosvenor and Scott showed that early adult-onset myopic eyes are expected to progress at a rate of $-0.18 \mathrm{D} /$ year compared to youth-onset eyes becoming myopic before 16 years of age who progressed at a rate of $-0.25 \mathrm{D} /$ year [5]. Jorge et al., conducted a study in the same setting between 2003 and 2006 and showed that at least $22 \%$ of 109 subjects with myopia between the age of 18 and 34 at recruitment time progressed by at least $0.50 \mathrm{D}$ over the 3 years [6]. Adult onset myopia also presents similar rates of change with about half of the subjects presenting an increase of $0.37 \mathrm{D}$ or higher over a 2 year period according to McBrien and Adams who studied a population of microscopists [27]. In the context of the Cambridge Anti-Myopia Study (CAMS), Allen et al. reported axial elongation of 0.15 $\pm 0.15 \mathrm{~mm}$ associated with an average progression of $-0.33 \mathrm{D}$ over a period of 2 years [28]. We report slightly lower values for a period of 4 years (2009-2013) in subjects of similar age. The CAMS results represent a good comparison term for the interpretation of our results as it reports on a clinical trial conducted in an atypical age range of 14-22 years of age, matching the profile of the preuniversity and university students as is the case of those reported 
by us during their myopia onset and progression. As expected values or myopia progression in these older populations are lower than those observed in children in other clinical trials [29].

We observed a small trend for axial length increase over the 4 years and being greater after lens discontinuation. However, part of this change might not be entirely attributed to actual elongation of the posterior pole. The change during and after treatment is minimal compared to the trend of progression before the treatment as shown in Table 1. An explanation can be found in the epithelial redistribution which decreases the central corneal thickness. As the IOLMaster measures axial length from the anterior corneal surface [30] corneal thickness changes should reflect in the biometric measurement. However, according to the refractive values involved (about $2.00-2.50 \mathrm{D}$ of myopia) this epithelial thickening is not expected to exceed the $20 \mu \mathrm{m}$ [31]. González-Mesa et al. reported changes in the axial length and in the anterior chamber depth in young adult subjects with stable myopia corrected with orthokeratology [32]. These changes reverted after lens discontinuation and their data are also in the range of $0.1-0.15 \mathrm{~mm}$ as presented in our subjects. Although we do not have baseline biometric values before lens fitting, the increase in axial length found after lens discontinuation might not reflect a true axial elongation after lens discontinuation.

Considering the above, a true stabilization effect by the corneal refractive therapy treatment is also plausible. Several studies, including two controlled and randomized clinical trials [16,17] have demonstrated that corneal refractive therapy is able to slow myopia progression by $40-50 \%$ in young children irrespective of their ethnicity or country in which they live. In those studies, the age of the children enrolled varied from 7 to 12 years of age at enrollment in the LORIC study conducted in Hong-Kong and published in 2005 [10] to 8-16 years of age in the study conducted by Kakita et al. in Japan and published in 2011 [12]. Regardless of the age range being different, both studies showed similar retention patterns. Furthermore, the Japanese study followed the subjects for five years, and the regulation effect, despite lower, continued over this period [33].

Considering the expected eye elongation is smaller in young adults with early adult-onset myopia than in eyes of children with early onset myopia, the potential benefit of regulating myopia in adult eyes will be lower. However, the present report, as well as other reports of myopia progression in adulthood reinforces the possibility that the strategies to regulate myopia progression investigated in children may also be effective later in life and it may be of value to regulate myopia progression in young adults by the use of corneal refractive therapy lenses.

Although the changes in the peripheral refraction pattern [19] are regarded as the mechanism to reduce myopia progression in corneal refractive therapy, this has not been fully validated. None of the studies has reported simultaneously the peripheral refraction changes to establish a direct and independent association that might point to peripheral refraction as a causative mechanism to regulate myopia progression in humans corrected with corneal refractive therapy. However, the results we present in Fig. 2, are consistent with the mechanism. In fact, despite the relatively low amounts of myopia corrected, both the tangential and sagittal focal lengths became significantly and slightly myopic respectively after the treatment while they were low myopic, emmetropic or significantly hyperopic before treatment. Radhakrisnan et al., in the context of the CAMS study, showed an association, although weak, between changes in peripheral refraction and myopia progression [34]. The nature of this report and the lack of a statistical approach to a larger population does not allow us to prove a relationship.

In summary, the present report of cases suggests that corneal refractive therapy can be also effective in decreasing myopia progression in university students presenting early adult-onset myopia. Clinical trials are needed in order to establish the veracity of this assumption as the present sample is too small to validate the use of orthokeratology as a strategy of myopia regulation in adultonset myopia. Such trials should also measure the benefit of helping to reduce in a clinically significant way the morbidity associated with myopia for those subjects.

\section{Disclosure}

The authors declare that they do not have any proprietary or financial interest in any of the materials mentioned in this article. CEORLab have received support in the past 5 years for research activities from companies with interest in myopia regulation treatments through the University of Minho. None of the authors holds interests in the instruments or devices mentioned in the present manuscript.

\section{Acknowledgement}

This study has been funded by FEDER through the COMPETE Program and by the Portuguese Foundation for Science and Technology (FCT) in the framework of projects PTDC/SAU-BEB/ 098391/2008, PTDC/SAU-BEB/098392/2008 and the Strategic Project PEST-C/FIS/UI607/2011.

\section{References}

[1] J.S. Pointer, A 6-year longitudinal optometric study of the refractive trend in school-aged children, Ophthalmic Physiol. Opt. 21 (2001) 361-367.

[2] S.M. Saw, W.H. Chua, C.Y. Hong, et al., Nearwork in early-onset myopia, Invest. Ophthalmol. Vis. Sci. 43 (2002) 332-339.

[3] D.I. Flitcroft, A model of the contribution of oculomotor and optical factors to emmetropization and myopia, Vis. Res. 38 (1998) 2869-2879.

[4] S.M. Saw, G. Gazzard, E.C. Shih-Yen, W.H. Chua, Myopia and associated pathological complications, Ophthalmic Physiol. Opt. 25 (2005) 381-391.

[5] T. Grosvenor, R. Scott, Three-year changes in refraction and its components in youth-onset and early adult-onset myopia, Optom. Vis. Sci. 70 (1993) $677-683$.

[6] J. Jorge, J.B. Almeida, M.A. Parafita, Refractive, biometric and topographic changes among Portuguese university science students: a 3-year longitudinal study, Ophthalmic Physiol. Opt. 27 (2007) 287-294.

[7] L.N. Davies, J.S. Wolffsohn, B. Gilmartin, Cognition, ocular accommodation, and cardiovascular function in emmetropes and late-onset myopes, Invest. Ophthalmol. Vis. Sci. 46 (2005) 1791-1796.

[8] F. Thorn, J. Gwiazda, R. Held, Myopia progression is specified by a double exponential growth function, Optom. Vis. Sci. 82 (2005) 286-297.

[9] C. Villa-Collar, J.M. Gonzalez-Meijome, Ortoqueratologia Nocturna, ICMColegio Nacional de Ópticos-Optometristas de España, Madrid, 2006.

[10] P. Cho, S.W. Cheung, M. Edwards, The longitudinal orthokeratology research in children (LORIC) in Hong Kong: a pilot study on refractive changes and myopic control, Curr. Eye Res. 30 (2005) 71-80.

[11] J.J. Walline, L.A. Jones, L.T. Sinnott, Corneal reshaping and myopia progression, Br. J. Ophthalmol. 93 (2009) 1181-1185.

[12] T. Kakita, T. Hiraoka, T. Oshika, Influence of overnight orthokeratology on axial elongation in childhood myopia, Invest. Ophthalmol. Vis. Sci. 52 (2011) 21702174.

[13] J. Santodomingo-Rubido, C. Villa-Collar, B. Gilmartin, R. Gutiérrez-Ortega, Myopia control with orthokeratology contact lenses in Spain (MCOS): study design and general baseline characteristics, J. Optometry 2 (2009) 215-222.

[14] J. Santodomingo-Rubido, C. Villa-Collar, B. Gilmartin, R. Gutiérrez-Ortega, Myopia control with orthokeratology contact lenses in Spain: refractive and biometric changes, Invest. Ophthalmol. Vis. Sci. 53 (2012) 5060-5065.

[15] L.E. Downie, R. Lowe, Corneal reshaping influences myopic prescription stability (CRIMPS): an analysis of the effect of orthokeratology on childhood myopic refractive stability, Eye Contact Lens 39 (2013) 303-310.

[16] P. Cho, S.W. Cheung, Retardation of myopia in orthokeratology (ROMIO) study: a 2-year randomized clinical trial, Invest. Ophthalmol. Vis. Sci. 53 (2012) 70777085.

[17] J. Charm, P. Cho, High myopia-partial reduction ortho-k: a 2-year randomized study, Optom. Vis. Sci. (2013) 530-539.

[18] J. Charm, P. Cho, High myopia-partial reduction orthokeratology (HM-PRO): study design, Cont. Lens Anterior Eye 36 (2013) 164-170.

[19] A. Queirós, J.M. González-Méijome, J. Jorge, C. Villa-Collar, A.R. Gutiérrez, Peripheral refraction in myopic patients after orthokeratology, Optom. Vis. Sci. 87 (2010) 323-329. 
G Model

CLAE 828 No. of Pages 6

6

J.M. González-Méijome et al./Contact Lens E' Anterior Eye xxx (2015) xxx-xxx

[20] T. Grosvenor, A review and a suggested classification system for myopia on the basis of age-related prevalence and age of onset, Am. J. Optom. Physiol. Opt. 64 (1987) 545-554.

[21] J.M. Gonzalez-Meijome, C. Villa-Collar, Nomogram, corneal topography, and final prescription relations for corneal refractive therapy, Optom. Vis. Sci. 84 (2007) 59-64.

[22] A. Queiros, C. Villa-Collar, J. Jorge, A.R. Gutierrez, J.M. Gonzalez-Meijome, Peripheral refraction in myopic eyes after LASIK surgery, Optom. Vis. Sci. 89 (2012) 977-983.

[23] D. Lopes-Ferreira, C. Ribeiro, H. Neves, M. Faria-Ribeiro, A. Queirós, C. VillaCollar, J. Jorge, J.M. González-Méijome, Peripheral refraction with dominant design multifocal contact lenses in young myopes, J. Optom. 6 (2013) 85-94.

[24] M. Faria-Ribeiro, A. Queiros, D. Lopes-Ferreira, J. Jorge, J.M. Gonzalez-Meijome, Peripheral refraction and retinal contour in stable and progressive myopia, Optom. Vis. Sci. 90 (2013) 9-15.

[25] J.M. González-Méijome, M.A. Faria-Ribeiro, D.P. Lopes-Ferreira, P. Fernandes, G. Carracedo, A. Queiros, Changes in peripheral refractive profile after orthokeratology for different degrees of myopia, Curr. Eye Res. (March) (2015) 1-9, doi:http://dx.doi.org/10.3109/02713683.2015.1009634 [Epub ahead of print].

[26] J.M. González-Méijome, S.C. Peixoto-de-Matos, M. Faria-Ribeiro, D.P. LopesFerreira, J. Jorge, J. Legerton, A. Queiros, Strategies to regulate myopia progression with contact lenses: a review, Eye Contact Lens (February) (2015) [Epub ahead of print].
[27] N.A. McBrien, D.W. Adams, A longitudinal investigation of adult-onset and adult-progression of myopia in an occupational group. Refractive and biometric findings, Invest. Ophthalmol. Vis. Sci. 38 (1997) 321-333.

[28] P.M. Allen, H. Radhakrishnan, H. Price, et al., A randomised clinical trial to assess the effect of a dual treatment on myopia progression: the Cambridge anti-myopia study, Ophthalmic Physiol. Opt. 33 (2013) 267-276.

[29] D.S. Fan, E.Y. Cheung, R.Y. Lai, A.K. Kwok, D.S. Lam, Myopia progression among preschool Chinese children in Hong Kong, Ann. Acad. Med. Singapore 33 (2004) 39-43.

[30] W. Haigis, Pseudophakic correction factors for optical biometry, Graefes Arch. Clin. Exp. Ophthalmol. 239 (2001) 589-598.

[31] A. Alharbi, H.A. Swarbrick, The effects of overnight orthokeratology lens wear on corneal thickness, Invest. Ophthalmol. Vis. Sci. 44 (2003) 2518-2523.

[32] A. Gonzalez-Mesa, C. Villa-Collar, A. Lorente-Velazquez, A. Nieto-Bona, Anterior segment changes produced in response to long-term overnight orthokeratology, Curr. Eye Res. 38 (2013) 862-870.

[33] T. Hiraoka, T. Kakita, F. Okamoto, H. Takahashi, T. Oshika, Long-term effect of overnight orthokeratology on axial length elongation in childhood myopia: a 5-year follow-up study, Invest. Ophthalmol. Vis. Sci. 53 (2012) 3913-3919.

[34] H. Radhakrishnan, P.M. Allen, R.I. Calver, et al., Peripheral refractive changes associated with myopia progression, Invest. Ophthalmol. Vis. Sci. 54 (2013) 1573-1581.

Please cite this article in press as: J.M. González-Méijome, et al., Stabilization in early adult-onset myopia with corneal refractive therapy,

Contact Lens \& Anterior Eye (2015), http://dx.doi.org/10.1016/j.clae.2015.06.009 